\title{
Understanding experiences of changing socioeconomic and psychosocial adversities during COVID-19 for freelancers working in the UK cultural sector: A rapid approach
}

\author{
Katey Warran*, Tom May, Daisy Fancourt, Alexandra Burton \\ Research Department of Behavioural Science and Health \\ Institute of Epidemiology \& Health Care \\ University College London \\ 1-19 Torrington Place, London, WC1E 7HB \\ *Corresponding author: k.warran@ucl.ac.uk
}




\begin{abstract}
There is a dearth of qualitative research exploring how freelancers working in the cultural industries have been affected during COVID-19. In particular, there is a lack of research exploring how socioeconomic and psychosocial adversities may have changed or evolved, and how these changes have been experienced. This study builds on qualitative interviews carried out in July-November 2020 ( $n=20$ ) by exploring findings from follow-up interviews conducted in May-July 2021 ( $n=16$ ). It presents a diversity of experiences, showing how some freelancers experienced small changes (e.g., to the kind of work carried out), with others experiencing major changes (e.g., leaving the sector completely). The study also explores experiences of ongoing or increased mental health impact, as well as changing attitudes to cultural work. It concludes with a call for highly bespoke support in the future that can address the huge disparity of experiences during this time.
\end{abstract}

\title{
Keywords
}

Freelancers, self-employed, COVID-19, qualitative, mental health, precarity 


\section{Introduction}

In response to the coronavirus-19 (COVID-19) pandemic, the cultural industries saw rapid and major changes in relation to how culture is produced and consumed. The first UK lockdown in March 2020 prompted the mass closure of arts and cultural venues and cancellation of inperson events, with venues then having to adhere to changing mandated restrictions as the pandemic continued. Many venues had to operate at reduced capacity, adapt their offering, or cease to operate at all. These changes fluctuated in line with the easing and tightening of social distancing throughout the pandemic, with many cultural venues oscillating between inperson and digital offerings, alongside hybrid models. The impact of these ongoing changes provided many challenges for the sector including fewer employment opportunities (Office for National Statistics, 2021), but also some opportunities including increased digital production (Radermecker, 2020), and some increases in home-based arts engagement (Bu et al., 2021).

A freelance career in the cultural sector has long been recognised as a path that may involve substantial financial or psychological risk, including periods of low or unpaid work, with social, cultural, and symbolic capital also important to 'making it' (Gerber, 2017; Menger, 2001). The conditions in which freelance cultural workers exist are precarious, as has been widely acknowledged within research in the last decade (Comunian \& England, 2020; Morgan \& Nelligan, 2017). However, the flux of the pandemic has exacerbated many of these preexisting challenges, particularly for those whose careers place in-person work at the heart of their offering, such as those that rely on live performances (Comunian \& England, 2020). Research conducted in 2020 showed that the first UK lockdown adversely affected employment and income for this group, with fewer opportunities available and income loss common (Creative Scotland, 2020; Cultivator, 2020; Oxford Economics, 2020; ScreenSkills, 2020). For example, survey results published in April 2020 exploring the impact of the pandemic on creative freelancers in Wales $(n=237)$ found that $85 \%$ of freelancers reported that their work either decreased sharply (25\%) or dried up completely (60\%) (Komorowski \& Lewis, 2020). Research also showed that employment precarity for freelancers during the first UK lockdown resulted not only in financial implications but negative consequences for mental health, social relationships, and sense of identity (May et al., 2020). This supports wider research showing a perceived link between financial hardship and lower wellbeing for performing arts professionals (Spiro et al., 2021).

Ongoing quantitative research has explored these work changes across different stages of the pandemic, for example showing how the numbers of freelancers has steadily declined since the onset of COVID-19 (Florisson et al., 2021). However, the nuances behind these changing employment numbers in relation to what work opportunities have reduced and why freelancers may choose to leave the sector is less known, with a dearth of research exploring the changing psychosocial impact of the pandemic on freelancers. This latter point is important because the complex 'social' aspects of a career in the cultural sector are viewed as essential 
for securing work opportunities through social networks, and contributing to perceived work enjoyment whereby work colleagues are often considered friends and sources of social support (Gerber, 2017; Lingo \& Tepper, 2013). Understanding how to support freelancers during the pandemic is underexplored, such as what strategies may be used to cope with work opportunity decline, changing social networks, and mental health impact. Given that major disruptions to work life can lead to serious mental health consequences (Marmot, 2005; Marmot et al., 2012), understanding the impact of ongoing disruptions is worthy of further attention.

Thus, given the rapid societal change taking place throughout the COVID-19 pandemic, it is important to explore how socioeconomic and psychosocial experiences may have evolved or changed for freelance cultural workers. It is also of interest to examine what specifically may have prompted particularly burdensome experiences throughout different pandemic stages (e.g., transitions in and out of multiple lockdowns), how these experiences have been perceived, and the consequences for mental health and wellbeing across time.

\section{Methods}

This research employed a longitudinal rapid appraisal qualitative approach, appropriate for examining processes of change. Rapid Appraisal is used to 'develop a preliminary, qualitative understanding of a situation' (Beebe, 1995, p. 42). It forms part of a wider selection of rapid qualitative approaches which are particularly useful for research that 'demands the sharing of findings in almost real time', whereby traditional qualitative methods would be too timeintensive (Vindrola-Padros et al., 2020, p. 2). Such approaches have been used in response to major societal changes such as during previous epidemics and, more recently, in response to the COVID-19 pandemic to explore healthcare delivery (Vindrola-Padros et al., 2020). Whilst rapid appraisal is a relatively new methodology, it's success in responding quickly to timesensitive topics and collating actionable findings quickly to decision makers is acknowledged (Taylor et al., 2018; Vindrola-Padros et al., 2020). The Rapid Appraisal approach enabled us to report our findings to Arts Council England to inform policymaking during the critical time of the pandemic.

The study involved two-stage interviews with freelancers working in the cultural industries, with the first interview taking place between July-November 2020 and the second interview taking place between May-July 2021. The first study explored participants' lived experiences of the pandemic and any socioeconomic and psychosocial impacts (May et al., 2020), with the current study focusing specifically on perceived changes in experiences and circumstances over time. The interview guide was adapted for the follow-up interview to ask about changes to the working and social lives of participants since the first interview, such as the impact of different stages of the pandemic on working conditions and job opportunities, as well as the impact of these changes on mental health. The interviews lasted an average of 
45 minutes and were conducted via either video or telephone call by researchers with backgrounds in social science (KW, TM).

\section{Participants}

20 self-employed freelancers working in the cultural sector from across the UK took part in initial interviews, with 16 of these freelancers participating in a follow-up interview. The remaining four participants were uncontactable following email invitations to participate. Convenience sampling was employed, with the study including people aged over 18 working within and across the performing arts, visual arts, and Film and TV industries, but care was taken to ensure a heterogeneous sample in terms of core demographics and profession (see May et al., 2020, for further information about sampling). Participation in the research was voluntary, and all participants were provided with details either verbally or in writing about the nature and purpose of the research and what their participation would involve. Ethical approval was provided by University College London research ethics committee [Project ID 14895/005] and all participants provided written informed consent. See Table 1 for demographic information.

Table 1: Characteristics of freelancers included in the follow-up interviews for this study

\begin{tabular}{|c|c|}
\hline Number of participants & 16 \\
\hline \multirow[t]{9}{*}{ Profession } & Contemporary Dance Teacher (1) \\
\hline & Film and TV Producer (2) \\
\hline & Independent Theatre Producer and Arts Administrator (1) \\
\hline & Musician (4) \\
\hline & Playwright/Theatre/Opera Director (3) \\
\hline & Independent Production Associate - arts and film (1) \\
\hline & Stage Actor (1) \\
\hline & Screen Actor (1) \\
\hline & Visual Artist (2) \\
\hline Age & $22-53(35.4)$ \\
\hline \multirow[t]{2}{*}{ Gender } & Male 5 \\
\hline & Female (11) \\
\hline \multirow[t]{4}{*}{ Ethnicity } & Black Other (1) \\
\hline & Indian (1) \\
\hline & Mixed other (1) \\
\hline & White \& Black Caribbean (2) \\
\hline
\end{tabular}


Analysis

As is typical of Rapid Appraisal, data collection and analysis took place concurrently (Beebe, 1995). This involved using RAP sheets (Rapid Assessment Procedure) after each follow-up interview to immediately document, and begin interpretation of, the interviews. The RAP sheet consisted of a table with thematic headings of interest based on the topic guide, along with a column for researcher notes, which was used to guide quick notetaking and systemisation of what was discussed. These RAP sheet summaries were then considered in regular team discussions ( $K W, T M$, and $A B$ ), alongside listening to relevant sections of the interview audio, to explore and map emerging findings. Key themes from the RAP sheets were then charted into a new table by $\mathrm{KW}$ to explore overarching thematic patterns which were discussed with the team ( $K W, T M$, and $A B$ ) and used to guide the write-up of the key findings. Given the way in which interviews were recorded and analysed in view of the rapid methodological procedure, the majority of the quotes included are paraphrased, rather than verbatim. However, some key quotes were transcribed verbatim and this is indicated throughout.

\section{Findings}

Four overarching themes and 11 sub-themes were constructed through the rapid assessment procedure. Together, the themes explore how experiences of socioeconomic and psychosocial adversities have changed over time, also unpacking the subjective implications of these adversities for the future, as shown in Table 2. 
Table 2. Themes and sub-themes from the rapid procedure, with descriptions of each subtheme

\begin{tabular}{|c|c|c|}
\hline Theme & Sub-theme & Description \\
\hline \multirow[t]{4}{*}{ Changing work life } & 1.1 Diversity of experiences & $\begin{array}{l}\text { Participants experienced a range of change - } \\
\text { from minor to major - depending on } \\
\text { circumstances }\end{array}$ \\
\hline & 1.2 Work patterns & $\begin{array}{l}\text { Changes were experienced in relation to the } \\
\text { kinds of work done, work lifestyle, and the } \\
\text { ways in which work opportunities were found }\end{array}$ \\
\hline & 1.3 Income streams & $\begin{array}{l}\text { Many relied on funding or supplementary work } \\
\text { to support income due to continued loss of } \\
\text { work }\end{array}$ \\
\hline & 1.4 Attitudes to work & $\begin{array}{l}\text { Many experienced a shift in perspective, with } \\
\text { work taking less priority and experiencing } \\
\text { changing ambitions }\end{array}$ \\
\hline \multirow[t]{2}{*}{ Changing social life } & 2.1 Shifting network connections & $\begin{array}{l}\text { Some personal connections were } \\
\text { strengthened, including domestic and } \\
\text { neighbourhood relationships. Other } \\
\text { connections, particularly work contacts, } \\
\text { became more peripheral }\end{array}$ \\
\hline & $\begin{array}{l}2.2 \text { Change in communication } \\
\text { methods }\end{array}$ & $\begin{array}{l}\text { Many moved away from digital } \\
\text { communications to either communicating less } \\
\text { within social networks or increasing in-person } \\
\text { contact }\end{array}$ \\
\hline \multirow[t]{3}{*}{$\begin{array}{l}\text { Ongoing mental } \\
\text { health and wellbeing } \\
\text { impact }\end{array}$} & 3.1 Psychological & $\begin{array}{l}\text { Some participants reported experiencing } \\
\text { ongoing and increased anxiety, depression, } \\
\text { stress, and reduced wellbeing, but some also } \\
\text { noted feeling positive about the future }\end{array}$ \\
\hline & 3.2 Physical & $\begin{array}{l}\text { Those who experienced adverse psychological } \\
\text { effects also experienced ongoing or new } \\
\text { physical symptoms, including stomach pains, } \\
\text { migraines, and lethargy }\end{array}$ \\
\hline & 3.3 Coping & $\begin{array}{l}\text { Changes to coping strategies were seen by } \\
\text { some, particularly in relation to reducing } \\
\text { activity-based coping and the introduction of } \\
\text { new self-help strategies }\end{array}$ \\
\hline \multirow[t]{2}{*}{$\begin{array}{l}\text { Perceived } \\
\text { implications for the } \\
\text { future }\end{array}$} & 4.1 Cultural sector - structural & $\begin{array}{l}\text { Similar to first interviews, participants reported } \\
\text { feeling structural problems will worsen in the } \\
\text { future e.g., inequalities, precarity }\end{array}$ \\
\hline & 4.2 Professional life & $\begin{array}{l}\text { Participants noted increased implications for } \\
\text { their professional lives, suggesting their lives } \\
\text { would not return to 'normal', expressing a } \\
\text { range of emotions about this }\end{array}$ \\
\hline
\end{tabular}




\section{Changing work life}

The extent to which participants' work lives changed since their first interview varied, with a diversity of experiences expressed (subtheme 1.1). Some participants reported only minor changes, stating that they were 'still at a standstill' (ID1), and that work life had 'not changed a great deal' (ID3), or 'not changed much' (ID22) since their first interview. Others described fluctuating changes during this period, with cultural work opportunities coming back after being 'non-existent last time' (ID5) and being like 'feast and famine' (ID20). A selection of participants reported major changes to their working lives, for example obtaining full-time employment in the arts and moving away from freelance work (ID9, ID21):

Yes, I have now started working full-time, which I would never do, if it wasn't for the pandemic. So, now, jumping from freelance, now I work 11 hours a day, five to six days a week... it's given me more thoughts about how much I don't want to work full-time, anywhere. (ID21, Film \& TV Producer, quote transcribed verbatim)

Others reported that they left the cultural industries completely, such as setting up their own business [not in the arts] (ID17), working in care (ID12) or teaching (ID11). For those who made these major changes, some reported considering staying in their new roles indefinitely (ID12, ID17), while others hoped to move back into an arts career, if they could secure arts project funding in the future (ID11).

The changes experienced were not just in relation to industry or job role, but also in relation to work patterns (subtheme 1.2). One change experienced was the type of work participants engaged in, relating to reduced options of cultural work since their last interview. One participant expressed letting their work as an artist 'drop off' to focus more on publishing because 'that's where the money is' (ID3). Even within publishing, the participant found the work to be less varied, with more content relating to the pandemic (ID3). This was similar to another participant who decided to focus on more 'business orientated' work (e.g., business plans, strategic planning) in place of face-to-face cultural work (ID4). A composer also expressed that there was 'less choice in the work overall' with more opportunities occurring in TV than in other industries (ID22), similar to another performer who previously worked across stage and screen who said their work had shifted to primarily being in TV and film (ID5).

Another dimension of changing working patterns was in relation to lifestyle. For their first interview, the majority of participants described working from home, with many using online methods to create and adapt their work, if opportunities were available at all. This time around, many had adopted hybrid approaches, working both in-person and online, with inperson work requiring adherence to COVID-19 social distancing guidelines which made work 'very strange' because 'so much of the relationship is withheld' (ID20). One participant (ID2) expressed how he felt the film \& TV industry were particularly regulated: 
I honestly say the film and TV industry was probably one of the most heavily, in a way, regulated with regards to protocols and guidelines, in regards to some other industries... So, we'd be working with masks all day, every day, you have temperature checks every day. Usually you're doing an at-home test before you come into work. (ID2, Film \& TV Producer, quote transcribed verbatim)

A number of participants also expressed growing tired of online working and trying to move away from it completely, reporting zoom fatigue (ID22, ID17, ID7, ID4) and noting the limitations of doing cultural work online because 'developing art is the opposite to preparing for a zoom meeting' (ID7). Whilst these challenges were mentioned in first interviews, they were reported to have intensified in the follow ups. However, one aspect of work that remained similar to the start of the pandemic was how work was secured. Prior to the pandemic, participants reported drawing on their social networks to find work, but said they had experienced a loss of this when the first UK lockdown began. This remained similar 8-12 months later, with many stating that new work opportunities were very limited and that social networks couldn't help because of limited opportunities, with many primarily surviving on postponed contracts from before the pandemic began (ID9, ID7). The exception to this was a performer who had found dramaturgical work through a professional contact (ID20).

Income streams also changed to varying degrees (subtheme 1.3). Previously, due to workload decline, income had been reduced for the majority of participants and many reported not being eligible for government funding. Whilst this was still the case for some participants (e.g., ID1, ID2, ID19), others found their workload and subsequently their income had started to return (e.g., ID5, ID3). Many also managed to balance small amounts of government support (e.g., the COVID-19 self-employment income scheme, and support from Arts Council England and Creative Scotland) or other support (e.g., charitable funds) with cultural work to try and acquire the level of income they had pre-pandemic (ID22, ID10). However, for some, limited or no funding combined with reduced cultural work opportunities meant having to take on supplementary work from outside of the cultural sector. This included working in supermarkets, recruitment, and doing administration or charity work (ID13, ID9, ID20). Others had to leave the sector completely due to financial concerns and limited funding (ID12, ID11). Several participants also noted that completing funding applications was akin to unpaid work; they required time and labour ('there's no time, they won't fill in the forms for you', ID12), with little feedback provided when applications were unsuccessful:

I've been putting in the odd application for Arts Council funding. It's just been six, eight months [of] rejections really on projects, and funding applications. So, yes, a fairly negative experience really... I was rejected... with not really any feedback, only that they preferred other applications. (ID19, Visual Artist, quote transcribed verbatim) 
One participant also secured project funding before the pandemic, but they 'lost the funding' (ID11) due to the challenges of executing it during COVID-19. They reported putting in 'so much planning' and 'never being paid for any of it' (ID11), thereby affecting the income they could have gained elsewhere.

The last sub-theme in this category involved changing attitudes to work (subtheme 1.4). Participants reported adopting a 'different perspective' (ID3), where 'time to think... fundamentally changed priorities' (ID9) and provided a new 'sense of purpose' (ID17). Whereas previously the focus of life was primarily on attaining and sustaining a career in the arts, focus shifted to home, family, friendships, and pets (ID9, ID11), with participants also reporting preferring a 'slower pace' to life that is 'less hectic' (ID3, ID11) where 'simple things' are viewed as 'important' (ID22). This shift was also, however, connected to reduced motivation to commit to working in the arts (ID9, ID19) - which was perceived as a precarious industry to continue working within - resulting in 'lost drive' and 'less ambition' (ID3). These changing attitudes were deeply intertwined with changing social life (theme 2), whereby the strengthening of ties to core relations such as partners and family facilitated a greater focus on personal life.

\section{Changing social life}

A diversity of change was experienced in relation to the social lives of participants, with many experiencing shifting network connections (subtheme 2.1), with some contacts becoming more peripheral in their networks and others moving more closely to the core. In terms of personal networks, the majority of participants reported the connections with people with whom they lived and those who lived locally becoming stronger. Changes in local connections were also described, with one participant using online networking to make new friendships in her local community. This translated into in-person relations, creating a 'change for the better', stressing a feeling of 'being more embedded in the community' (ID3). Another participant noted feeling 'happy in their neighbourhood network' (ID21), but also 'a bit exploited' by neighbours who they had helped out during lockdowns (ID21).

In terms of professional networks, participants reported the loss of their professional networks in their first interview due to being unable to meet colleagues in-person during the first UK lockdown, with many of these colleagues also considered as friends. For those who experienced a changing attitude to work, this loss was sustained and, for some, intensified. This resulted in a strengthening of their personal relations, while work colleagues and those who did not live locally became more peripheral in their network. Participants reported 'engaging more with family' (ID1, ID3), and seeing work colleagues less (ID22, ID1, ID19, ID11, ID21), resulting in 'smaller social circles' (ID9), 'feeling cut off' (ID11), and limited socialising (ID21, ID7). Many also reported feeling closer to their romantic partners (ID19, ID1, ID22, ID9, ID21) who were identified as a key source of social support. For example: 
I didn't really need other people, because I really enjoyed the relationship with my boyfriend and the company, the partnership that we've shared... So, I wasn't lonely in the pandemic, and I think in comparison to many other freelancers, who are single, it made a massive difference that I had all my social needs covered. Because I lived with my favourite human and my best friend, who is also my boyfriend, so, I think, in terms of social needs, I had all of them covered. (ID21, Film \& TV Producer, quote transcribed verbatim)

However, other participants increased their social network through networking online with others in the cultural industries (ID13) and reported strengthened colleague relations due to a sense of shared experience of pandemic-related challenges and 'time to talk' (ID20). The opportunity to meet in person when restrictions eased was viewed as a way to 'socialise' (ID5) 'come together' and 'cement' work connections (ID10), with honesty, vulnerability and shared experience viewed as facilitative of this coming together (ID7, ID13, ID21). These shifts were, however, described as complex, as the pandemic 'strengthened the friendship aspect of some arts connections and weakened others' (ID3), with work relations viewed as 'up and down' (ID5). Further, the sense of connection to arts organisations was considered to have weakened, with participants feeling let down by organisations ('feeling sort of thrown out', ID1) due to 'lack of contact' and a feeling of 'organisations versus freelancers' (ID7). Namely, there was a sense that there was a 'divide in the community' where arts organisations were 'not considering freelancers' (ID7).

Many participants experienced a change in communication methods (subtheme 2.2) within their social networks. For example, a number of participants actively reduced their engagement in digital communication since their first interview. This included decreasing their use of social media such as Twitter (ID5, ID9, ID3, ID12), as well as online communication tools (notably Zoom) for connecting with both personal and professional contacts (ID7, ID22, ID21, ID17). As one participant explained:

When it comes to friends, I prefer to call them, more meaningful then... I really don't like reconnecting with people online, so that doesn't really work for me, so, I just didn't do it. (ID21, Film \& TV Producer, quote transcribed verbatim)

Social media was also seen as 'divisive' and 'fragmented', with organisations and individuals permitting a message 'that everyone is back at work in the arts' (ID9), when 'it's only half the workforce' (ID12). Further, several participants reported starting in-person work which resulted in communicating with others in studios (ID3, ID22, ID12), on film sets (ID5, ID20), and at outdoors gigs (ID13), alongside seeing family and friends in-person, such as at restaurants (ID13). Nevertheless, some participants reported a continuation of spending more time at home than before the pandemic and feeling reluctant about meeting up with others in person. One participant continued to avoid socialising in pubs because they had 'lost the habit' (ID17), another found these situations 'overwhelming' (ID20), and another didn't want to socialise 
because of fear of catching COVID-19 (ID12). This latter point is connected to the perceived impact of the pandemic on mental health and wellbeing (theme 3), whereby several participants reported feeling lonelier since the start of the pandemic, alongside losing the desire to socialise in person when restrictions permitted them to do so (ID11, ID9, ID20).

\section{Ongoing mental health and wellbeing impact}

Several participants reported an increase in stress and anxiety in their first interview, and this was found to be further exacerbated during subsequent lockdowns for some, often manifesting as psychological symptoms (subtheme 3.1). This included an increase in anxiety (ID20, ID22, ID12), panic (ID1), stress (ID12, ID11, ID21, ID5), depression (ID20), rumination (ID20), a feeling of the mind racing (ID9), claustrophobia (ID10), reduced self-esteem and confidence (ID12, ID9), and reduced overall wellbeing and quality of life (ID20, ID3, ID9, ID11). One participant also reported feeling suicidal 'last year' (in 2020) but, having since secured a job outside of the cultural industries, described starting to feel more positive about the future (ID12). Many also reported ongoing existential questioning relating to their sense of identity, progression, and purpose (ID12, ID1, ID20, ID2). For some, this resulted in feeling hopeless (ID1, ID7), out of control (ID12, ID1), and finding it hard to discern time during periods of no work (ID7). The lack of work was also perceived as 'heart-breaking' (ID11, ID21), and left one feeling that 'it doesn't matter if I just go to bed' (ID20), with another expressing that the lack of funding received for work was 'demoralising' (ID19). These psychological experiences also connected to a range of moods, including feeling guilty (ID1), low (ID2, ID3), worried (ID9, ID13, ID21), devastated and bereaved (ID7), shameful (ID9), sad (ID20, ID9), paralysed (ID20), lost (ID10), depleted (ID11), and, in relation to the lack of support from government, betrayed (ID11).

Nonetheless, for several participants their negative affect was also experienced alongside more positive feelings, with the pandemic period described as a 'rollercoaster of emotions' (ID3). This meant that negative feelings 'came in waves' (ID9), with feelings such as being 'excited' about the future (ID9), 'positive' since work returned (ID2), and 'thrilled' about future projects (ID7) also part of this 'turbulent' time (ID3). Moreover, these positive feelings increased for some as work opportunities returned, with one individual reporting feeling 'better over time' (ID5) and suggesting that they had become 'more resilient' through the pandemic (ID11) and had 'not given up the joyful, creative part of myself' (ID21). One participant who had left the cultural industries to start their own business also reported enjoyment from doing so:

Yeah well I've started my own business since I last spoke to you... And just really love it, really enjoyed it found it really rewarding, and it just confirmed that I wanted to do more work around that. (ID17, Independent Production Associate (Arts \& Film), quote transcribed verbatim) 
The negative aspects of this turbulence, however, had a dramatic effect on some, also displaying as physical symptoms (subtheme 3.2). Whilst in their first interview some physical symptoms were reported (e.g., sleep disturbances), these were exacerbated for some, with new symptoms also occurring. One participant (ID1) reported that an instance since their first interview where they couldn't see their newborn niece due to a change in social distancing restrictions led to a feeling of their chest tightening:

Not even to hold her, but just to see her at all. But then I wasn't allowed. I think it was three weeks after that, oh, I just wasn't at a good place. There was one day, I was so annoyed that I was just crying all night, basically. Not upset crying, just complete meltdown crying... My chest would get really tight. (ID1, Musician, quote transcribed verbatim)

Two participants described ongoing stomach pains (ID5, ID9), with one (ID9) describing these as 'IBS bouts' that they didn't have before the pandemic. Another said that their physical symptoms had continued to increase since their first interview and included 'heart thumping, sweaty attacks' and 'a mild electric shock feeling' that also 'affected memory really badly' (ID12). The increased usage of Zoom also led to a new 'physical reaction' for one participant, making her feel sick (ID7). In relation to sleep, several reported not sleeping well (ID10, ID12, ID9), or feeling lethargic (ID7, ID9, ID12), but one participant said their sleep had improved since their first interview. This participant had moved to a new house to remove 'environmental stressors' (ID22).

A number of participants also reported changes to their coping strategies (subtheme 3.3) since their first interview. Several described feeling that they had reached a peak of recreational activities and were no longer experiencing these activities as protective for their mental health, as reported in their first interview. One participant shared that there is 'only so much yoga and baking you can do' (ID10), with another saying that the 'novelty' of ordering food and subscriptions online 'ran out' (ID21), and others reducing activities such as knitting and sewing (went into a 'knitting frenzy'; 'half done sewing projects'; 'slowed up', ID20), mindfulness apps ('using headspace less now', ID9), exercise (ID13), and yoga (ID3). However, a number of participants also described continuing to use coping strategies such as sea swimming (ID1) and walking (ID22, ID17, ID3, ID9), reporting that they were still providing support. Further, a number of participants said that they had actively reduced their intake of social media, the news and TV to support their mental health (ID5, ID9, ID3, ID12).

Participants also described using new self-help strategies that were beneficial such as engaging in developing their self-awareness (ID17), goal setting (ID20), and diary writing:

I've been keeping a diary, and I stopped doing that a few weeks ago. But from the beginning of January up until maybe halfway through April, I was writing a diary every day. Not a big long thing of what l'd done the day, but just bullet points, just to write it down on a page. 
Because I was saying last time, I think, to [other researcher], as well, I was just losing track of time. There were times I just didn't know what day it was. (ID1, Musician, quote transcribed verbatim)

Several also drew upon formal support. One participant started medication for their mental health when they 'realised the pandemic would go on' which supported them in having 'energy to fill in job applications for other work' (ID12). Others described continuing with therapy sessions which were 'amazing support' (ID3), or finishing therapy but continuing to use 'the tools learned through it' (ID22). Finally, some participants were avoidant in their coping ('trying not to think about the future', ID3) while others were accepting of the challenges brough on by the pandemic ('accept it', ID1; 'making it work', ID13).

\section{Perceived implications for the future}

Many participants struggled to articulate the implications of adversities for the cultural sector because of the perceived uncertainty of the future ('in the waiting room', ID7). However, certain structural problems were highlighted (subtheme 4.1).

Firstly, participants identified implications for the workforce in the cultural sector. There was a sense that the pandemic had exacerbated job precarity, and that the future would mean 'less jobs' (sic) (ID7), with 'commercial jobs the only ones left' (ID21) making work in the cultural sector 'unsustainable' (ID10). These views were similar to that expressed in the first interviews. However, there was now a feeling that the government would continue to be 'a bit blind to the self-employed', overlooking the value that freelancers contribute to the cultural workforce (ID19). Further, building on the fears expressed in their first interviews, participants' concern about diversity in the sector remained, with some noting that the future will be 'terrible for diversity' and the 'workforce will change' (ID9). Further, this time there was an increased concern that many will leave the sector, if they have not done so already, with participants fearing that the sector will 'lose a lot of world-class artists' (ID7), with many looking at 'non-arts' and 'community' opportunities (ID1, ID3, ID13).

Another aspect of these structural implications concerned the kinds of culture that might be sustained and the patterns of cultural work. Participants thought that the 'big shows will be up on their feet first' (ID9) and that there will be 'reduced capacity' and 'more restrictions' in the future at cultural production sites (ID7). In addition, there was a feeling that there would be a continued 'increase in home working' (ID3) and an increase in 'online working' (ID19) and 'YouTube and TV' opportunities (ID13), with concerns that the latter would not be paid well. Several also felt that PPE (personal protective equipment) may be needed at work for some time: 
I think COVID and health and safety regarding COVID will definitely stay around for a couple of years. I'm very much prepared for always having a mask in my bag at this point as a standard piece of equipment as I would with anything else, just like work shoes. (ID2, Film \& TV Producer, quote transcribed verbatim)

This complemented the feeling of several participants who noted that the live performance industries - notably live music - would continue to be the most affected, in contrast to the Film \& TV industries (ID5, ID1). Relatedly, it was perceived that current funding application processes needed to change, in view of the aforementioned time-intensive nature of them (ID12), with participants also concerned about funding cuts (ID13). These funding issues were acknowledged as existing before the pandemic, but as persisting and growing since the pandemic's onset, thereby having implications for who can work in the cultural sector in the future and the type of work that is funded. One participant commented that the lessons learned from the pandemic could be positive for the sector, but 'money would be needed to implement changes' (ID10).

Moreover, these structural issues were discussed in light of wider socioeconomic changes in relation to the impact of Brexit and changing socio-political values, which were perceived by one participant as now 'less compassionate' and 'harsher' than prior to COVID19 (ID12). These societal changes were viewed as contributing to the precarity of the cultural sector.

Participants also noted potential implications for their own individual professional lives (subtheme 4.2). Firstly, there was a sense that work life 'won't go back to normal' (ID9) and that the future will be a case of 'surviving and not thriving' (ID11). In addition, one participant said that they will 'live life closer' in relation to working more locally in the future. Others also commented on social changes to their future work life, such as 'working with others more intimately' (ID1) and 'making more time for people' (ID5). A range of emotions were also expressed in relation to thinking about the future, with some 'hopeful' (ID1, ID5) and 'optimistic' (ID2) and others unable to see any 'prospects' or 'positives' to the changes to their career (ID20).

Interestingly, despite many worrying about the future of their professional lives in the cultural sector, a number of participants said that they would continue to pursue a career in the cultural industries in the future (ID1, ID5, ID9, ID13, ID12). Several also connected this to their identities, stating that 'staying in the arts is not a choice' (ID11) because it's 'integral' (ID1) to who they are.

\section{Discussion}

This study aimed to explore how individual socioeconomic and psychosocial experiences have changed or evolved for freelancers working in the cultural industries since the start of the COVID-19 pandemic in the UK. It shows how ongoing adversities manifested as changes to 
work life, with some experiencing small changes (e.g., to the kind of work carried out) and others experiencing major changes (e.g., leaving the sector to pursue alternative employment), as well as varied changes to social lives. The study also identified how some participants experienced ongoing or increased negative mental health impacts, manifested as psychological and physical symptoms, and that participants perceived there are, and will continue to be, implications of the pandemic for the cultural sector (e.g., worsening structural problems) and their working lives.

This study supports previous research that suggests the pandemic is exposing and widening existing inequalities, despite initial claims that the pandemic was a national and global collective experience (Wright et al., 2020). Notably, adversities relating to loss of employment, reduced income, and a lack of access to financial support have been experienced among some groups more than others (Wright et al., 2020), and our research shows that cultural freelancers are one of the professional groups particularly badly affected. For example, our initial research showed that inconsistent government support meant that while some creative freelancers received financial support, others in unstable freelance job situations (e.g., those new to working in the cultural industries with less social and symbolic resources than those who are more established) or those with portfolio careers (e.g., balancing PAYE and noncultural work with freelance cultural work) were less likely to receive funding. Although small scale, our new findings build on this to show that those who began the pandemic in an insecure position continued to experience increasing hardship as the pandemic continued, as has been acknowledged elsewhere (Banks \& Connor, 2020). Participants who did not receive adequate funding initially or who were ineligible (the ones also more likely to be already working within a landscape of uncertain job prospects) were those who either left their artistic career to work in a different sector or shifted the focus of their work into a different role within the cultural industries, experiencing mental health consequences as a result. Even when small amounts of funding or financial support were acquired later and combined with supplementary work, these adversities were too great to sustain their previous freelance cultural careers. Whilst this is a complex picture as change in career choice was also connected to the industry worked within and the kind of work done, there is an indication here that the pandemic has exacerbated pre-existing inequalities in the sector (see Brook et al., 2020).

Thus, whilst a freelance career may seem like an occupation that will always entail uncertainty with regards to job security, the extent of this insecurity varies considerably, and funding structures could have acknowledged this further. ${ }^{1}$ These nuances can be unpacked in light of the conceptualisation of precarity as put forward by Rodgers and Rodgers (1989) and discussed in relation to the cultural industries and the COVID-19 pandemic by Comunian \& England (2020). Four dimensions are outlined in relation to uncertainty: length of employment; control over work; regulatory frameworks for the sector; and wages. The amount of financial

\footnotetext{
${ }^{1}$ Whilst it was recognised in Scotland that freelancers needed extra financial support (e.g., Creative Scotland Hardship Funding), specific funds for freelancers have been limited elsewhere in the UK.
} 
and social support required for each freelancer working in the cultural industries will vary depending on which combinations of these different dimensions exist, and how they are experienced and negotiated at an intersubjective level. Further, these different dimensions and experiences have varied during different stages of the pandemic, as our research shows. During times of 'opening up' after lockdowns, for example, precarity varied dramatically for freelancers because only certain people were able to transition back into in-person work (e.g., those working in Film \& TV), with many remaining in a state of cultural work stasis (e.g., theatrical performers).

Accordingly, another reflection from this study is the way in which different industries have been affected. In our initial research, whilst experiences of adversities did vary, the issues described were primarily in relation to having no in-person work and the challenges of online working. Our study shows how these circumstances changed for people working in certain industries. As elucidated by Walmsley, Gilmore \& O'Brien (2021), Film, TV, and publishing sectors are showing signs of 'recovery and growth', but other industries, particularly the performing arts who have experienced uncertainty around when and how to open up, are still struggling. Our study supports this claim.

Further, whilst attitudes towards online working varied when we first spoke to participants and this was still the case to some extent in our follow-up interviews, we saw an increase in frustration. This was even the case for work that was more suited to online platforms (e.g., publishing, teaching). This raises questions about whether 'going online' is a viable option for certain industries where in-person aspects have traditionally been focal, as has been highlighted in wider research. Spiro and colleagues $(2021$, p.13) found that, for those working in the performing arts during the first UK lockdown, adapting work online was not always possible and was a 'poor substitute for face-to-face activities'. Research on the impact of the pandemic on arts residencies also shows a similar pattern to our findings; in a first survey conducted in May-June 2020, results showed that $40 \%$ of respondents felt virtual residencies were not feasible (Res Artis and UCL, 2020). Then, in their second survey (Nov 2020-Jan 2021), qualitative findings showed that many desired an experience that could take them away from the screen, also expressing hesitancy about digital opportunities (Res Artis and UCL, 2021).

In view of the challenges of online working, a range of reports seeking to inform organisational strategy or policymaking in the future have suggested that resources are needed to support with how to move cultural events and workshops online (Culture Counts, 2020), provide opportunities for freelancers to upskill including digital training (ScreenSkills, 2020), and conduct research to identify and remove 'barriers to entry into the digital market' (Department for Digital Culture Media \& Sport; \& Arts and Humanities Research Council, 2021). However, this study suggests that we not only need to answer the question of how to support this workforce to 'go digital' but also whether we should be doing so. If there are certain kinds of cultural production (notably, performing arts production) that are not so well suited to online delivery, then future focus should also be on alternative ways of working that are 
innovative and economically viable at times when traditional ways of engaging (such as inperson at concert halls, theatres, and stadiums) cannot happen. This is a challenge, but there has been a selection of audio-based, socially distanced, and 'blended' projects since the pandemic began, which suggests that there may be innovative options to develop and broaden the concept of 'the digital' in the future (Child et al., 2021). Viewing the rapid increase of mental health impact explored in this study alongside the decline of autonomy in relation to kinds of work and expressed changing attitudes in relation to digital working, finding new offline or 'blended' ways of working may also be vital to ensure a motivated and healthy freelance workforce in the future.

The changing attitude to work experienced by some is also an interesting finding. There is a wealth of literature published pre-pandemic showing that many pursue a cultural career (particularly artists) because it is akin to a vocational calling (Gerber, 2017), describing their work as satisfying or pleasurable (Bain, 2005). Further, this connects to discussions concerning artistic identity, where being an artist is viewed as providing meaning and purpose (Lingo \& Tepper, 2013), even considered a 'sacred' profession (Simpson, 1981) providing 'a necessary ritual for artistic self-actualization' (Hooks, 1995, p. 128, as cited in Bain, 2005). Thus, the dramatic shift, by some, to move away from a career that was once at the heart of who they were feels surprising. Looking to the wider literature on career transitions, changes to identity as a result of these transitions have been viewed as both positive (growth experiences) and negative, such as in relation to detrimental health repercussions (Oakland et al., 2013). The picture presented in this study, however, is not clear. On the one hand, we found a range of adverse mental health consequences with some unable to see their career shifts as 'positive'. But, on the other hand, many reported finding meaning in new ways, with their relationships with certain people (such as romantic partners) flourishing. We cannot make a normative assumption about a career in the cultural industries for this group as the only source of wellbeing nor suggest all participants 'should' try to get their pre-pandemic work lifestyles back should society return to a state of 'normality' in the future (i.e., when cultural production sites can operate without any restrictions). Indeed, for some, their newfound directions seemed to provide a subjective fulfilment that they did not have before.

In fact, those who did not renegotiate their identities may be putting themselves in a psychologically more vulnerable position because viewing their cultural careers as 'not a choice' could mean continuing to pursue their career in turbulent conditions, even if it could cause self-harm. Further, those people whose working lives are not 'back to normal' may also suffer mental health distress from the idealised image that the future will mean 'building back better' as per government rhetoric. Some cultural organisations and individuals publicly sharing their success stories of 'going back' online, such as via social media, may present an image of growth and positivity as the norm, when a large proportion of freelancers, and the sector at large, are still acutely experiencing the pandemic's impact. This combination of the individual's commitment to achieving a cultural career despite adversities and the presentation 
of opportunities available builds on Menger's (2014) theory of art and achievement under uncertainty. Whilst uncertainty can fuel innovation, it can also present a delusion because the prospect of achievement in the cultural industries is enough to motivate individuals to continue to pursue their careers, even if there are very few opportunities and the likelihood of 'making it' is minimal. Thus, the construction of 'post-lockdown' success shared by major arts and cultural organisations may provide hope for the individual freelancer that their pursuits are worthwhile, when opportunities may be limited. Nevertheless, it remains for future research to explore this further, in line with the changing social conditions of COVID-19 and the opportunities available.

\section{Conclusion}

The findings of this study are rooted in a foundation of diverse experiences, whereby the adversities of the pandemic affected freelancers in the cultural industries in a range of ways based on economic, social, and psychological factors. Thus, the future support provided for this group needs to be highly bespoke, considering not only loss of income, but the specific occupation and industry worked within, what dimensions of precarity have been experienced, and how they have been experienced, as well as what the mental health impact of the pandemic has been. Moreover, the complex factors that combine together to lead some to continue their freelance careers, some to shift their artistic focus, and others to leave the sector entirely are also worthy of following in greater detail in future research as the pandemic continues, unpacking further the inequality of experience that is embedded in the impact of COVID-19.

\section{Acknowledgments}

The authors would like to thank Dr Cecilia Vindrola for her guidance on using Rapid Appraisal. The authors would also like to thank the participants who gave their time to be interviewed for the study.

\section{Funding statement}

This follow-up study was funded by Arts Council England [INVF-00404362]. The initial study and data came from the COVID-19 Social Study which is funded by the Nuffield Foundation [WEL/FR-000022583]. The study was also supported by the MARCH Mental Health Network funded by the Cross-Disciplinary Mental Health Network Plus initiative supported by UK Research and Innovation [ES/S002588/1], and by the Wellcome Trust [221400/Z/20/Z]. DF was funded by the Wellcome Trust [205407/Z/16/Z]. 


\section{Declaration of interest statement}

The authors declare no potential conflicts of interest with respect to the research, authorship and/or publication of this article.

\section{References}

Bain, A. (2005). Constructing an artistic identity. Work, Employment and Society, 19(1), 2546. https://doi.org/10.1177/0950017005051280

Banks, M., \& Connor, J. O. (2020). "A Plague upon Your Howling": art and culture in the viral emergency. Cultural Trends, 0(0), 1-16. https://doi.org/10.1080/09548963.2020.1827931

Beebe, J. (1995). Basic Concepts and Techniques of Rapid Appraisal. Human Organization, 54(1). https://www-jstororg.libproxy.ucl.ac.uk/stable/44126571?seq=1\#metadata_info_tab_contents

Brook, O., O'Brien, D., \& Taylor, M. (2020). Culture is bad for you. Manchester University Press. https://manchesteruniversitypress.co.uk/9781526144164/

Bu, F., Mak, H. W., Bone, J. K., \& Fancourt, D. (2021). Longitudinal changes in home-based arts engagement during and following the first national lockdown due to the COVID-19 pandemic in the United Kingdom. MedRxiv, 2021.05.14.21257233. https://doi.org/10.1101/2021.05.14.21257233

Child, D., Gray, K., Weeks, H., \& Wright, J. (2021). Why digital isn't enough . Arts Professional. https://www.artsprofessional.co.uk/magazine/article/why-digital-isnt-enough

Comunian, R., \& England, L. (2020). Creative and cultural work without filters: Covid-19 and exposed precarity in the creative economy. Cultural Trends, 29(2), 112-128. https://doi.org/10.1080/09548963.2020.1770577

Creative Scotland. (2020). COVID-19 Scottish Creative Sectors Survey / Creative Scotland. https://www.creativescotland.com/resources/professional-resources/research/creativescotland-research/covid-19-scottish-creative-sectors-survey

Cultivator. (2020). Covid-19 Survey for Creative and Cultural Sectors in Cornwall and Isles of Scilly.

Culture Counts. (2020). Covid-19 Impact on the Cultural Sector: Snap Survey Results.

Department for Digital Culture Media \& Sport; \& Arts and Humanities Research Council. (2021). Boundless Creativity: Culture in a time of COVID-19.

Florisson, R., O’Brien, D., Taylor, M., McAndrew, S., \& Feder, T. (2021, March). The impact of Covid-19 on jobs in the cultural sector - part 3. Centre for Cultural Value. https://www.culturehive.co.uk/CVIresources/the-impact-of-covid-19-on-jobs-in-thecultural-sector-part-3/

Gerber, A. (2017). The work of art: value in creative careers. Stanford University Press. Hooks, B. (1995). Art On My Mind: Visual Politics. The New Press. 
Komorowski, M., \& Lewis, J. (2020). The Covid-19 Self-Employment Income Support Scheme: How will it help freelancers in the creative industries in wales?

https://www.gov.uk/guidance/claim-a-grant-through-the-

Lingo, E. L., \& Tepper, S. J. (2013). Looking Back, Looking Forward: Arts-Based Careers and Creative Work. Work and Occupations, 40(4), 337-363.

https://doi.org/10.1177/0730888413505229

Marmot, M. (2005). Social determinants of health inequalities. Lancet (London, England), 365(9464), 1099-1104. https://doi.org/10.1016/S0140-6736(05)71146-6

Marmot, M., Allen, J., Bell, R., Bloomer, E., \& Goldblatt, P. (2012). WHO European review of social determinants of health and the health divide. The Lancet, 380(9846), 1011-1029. https://doi.org/10.1016/S0140-6736(12)61228-8

May, T., Warran, K., Burton, A., \& Fancourt, D. (2020). Socioeconomic and psychosocial adversities experienced by creative freelancers in the UK during the COVID-19 pandemic: A qualitative study (pre-print). SocArXiv. https://doi.org/10.31235/OSF.IO/74SDR

Menger, P. M. (2001). Artists as workers: Theoretical and methodological challenges. Poetics, 28(4), 241-254. https://doi.org/10.1016/S0304-422X(01)80002-4

Morgan, G., \& Nelligan, P. (2017). The Creativity Hoax. The Creativity Hoax. https://doi.org/10.2307/J.CTT1Z27HWJ

Oakland, J., MacDonald, R., \& Flowers, P. (2013). Identity in crisis: The role of work in the formation and renegotiation of a musical identity. British Journal of Music Education, 30(2), 261-276. https://doi.org/10.1017/S026505171300003X

Office for National Statistics. (2021). Vacancies and jobs in the UK - Office for National Statistics.

https://www.ons.gov.uk/employmentandlabourmarket/peopleinwork/employmentande mployeetypes/bulletins/jobsandvacanciesintheuk/april2021

Oxford Economics. (2020). The Projected Economic Impact of COVID-19 on the U.K. Creative Industries. https://www.oxfordeconomics.com/recent-releases/The-ProjectedEconomic-Impact-of-COVID-19-on-the-UK-Creative-Industries

Radermecker, A.-S. V. (2020). Art and culture in the COVID-19 era: for a consumer-oriented approach. SN Business \& Economics 2020 1:1, 1(1), 1-14.

https://doi.org/10.1007/S43546-020-00003-Y

Res Artis and UCL. (2020). COVID-19: Impact Survey on the Arts Residencies Field Survey I of III.

Res Artis and UCL. (2021). COVID-19: Impact Survey on the Arts Residencies Field Survey II of III.

Rodgers, G., \& Rodgers, J. (1989). Precarious Jobs in Labour Market Regulation: The Growth of Atypical Employment in Western Europe. International Labour Organisation.

ScreenSkills. (2020). What can we do to help? Freelance survey. 
Simpson, C. R. (1981). Soho: The Artist in the City. University of Chicago Press.

Spiro, N., Perkins, R., Kaye, S., Tymoszuk, U., Mason-Bertrand, A., Cossette, I., Solange, G., \& Williamon, A. (2021). The Effects of COVID-19 Lockdown 1.0 on Working Patterns, Income, and Wellbeing Among Performing Arts Professionals in the United Kingdom (April-June 2020). Frontiers in Psychology, 11, 4105.

https://doi.org/10.3389/fpsyg.2020.594086

Taylor, B., Henshall, C., Kenyon, S., Litchfield, I., \& Greenfield, S. (2018). Can rapid approaches to qualitative analysis deliver timely, valid findings to clinical leaders? A mixed methods study comparing rapid and thematic analysis. BMJ Open, 8(10), 5-7. https://doi.org/10.1136/bmjopen-2017-019993

Vindrola-Padros, C., Chisnall, G., Cooper, S., Dowrick, A., Djellouli, N., Symmons, S. M., Martin, S., Singleton, G., Vanderslott, S., Vera, N., \& Johnson, G. A. (2020). Carrying Out Rapid Qualitative Research During a Pandemic: Emerging Lessons From COVID-19. Qualitative Health Research, 104973232095152. https://doi.org/10.1177/1049732320951526

Wright, L., Steptoe, A., \& Fancourt, D. (2020). Are we all in this together? Longitudinal assessment of cumulative adversities by socioeconomic position in the first 3 weeks of lockdown in the UK. Journal of Epidemiology and Community Health, 74(9), 683-688. https://doi.org/10.1136/jech-2020-214475 